Int. J. Dev. Biol. 50: 571-573 (2006)

doi: $10.1387 / \mathrm{ijdb} .052132 \mathrm{ba}$

Short Communication

\title{
Prevalence and genetics of immotile-cilia syndrome and left-handedness
}

\author{
BJORN A. AFZELIUS*,1 and UNNE STENRAM ${ }^{2}$ \\ ${ }^{1}$ Department of Zoophysiology, Arrhenius Laboratories F3, Stockholm University, Sweden and ${ }^{2}$ Department of Cytology, \\ Lund University, University Hospital, Lund, Sweden
}

\begin{abstract}
Immotile-cilia syndrome is characterized by severe respiratory distress from early infancy, and also often by situs inversus. The first description of the disease was based on just four persons, but reasons were given to suggest that the disorder may not be exceedingly rare. The purpose of the present study was to estimate just how rare or how common it is and to evaluate its association with situs inversus and with left-handedness. Data were mainly obtained from contacting a large number of Swedish clinicians who kindly informed us about their patients with suspected immotile-cilia syndrome. Diagnosis was in most cases performed by electron microscopical examination of nasal cilia or of spermatozoa. Based on these data, the prevalence of the syndrome in Sweden with or without situs inversus was estimated to be not far from 1 in 10,000 . The syndrome consists of several subgroups that have a randomized determination of situs asymmetry ( $50 \%$ of these have situs inversus) and one subgroup in which situs inversus is not found. This results in a frequency of situs inversus in the syndrome of about $44 \%$. Left-handedness is no more common than it is in healthy persons and no more often associated with situs inversus than with situs solitus. In all cases it is about $14 \%$. It is concluded that the two major anatomical/ physiological asymmetries of the human body are found with frequencies which indicate that they develop independently of each other. Both conditions appear with prevalences that may have changed at a secular scale, left-handedness with a substantial increase and situs inversus with a less dramatic increase.
\end{abstract}

KEY WORDS: primary ciliary dyskinesia, PCD, Kartagener syndrome, situs inversus, left-handedness

Immotile-cilia syndrome - also named primary ciliary dyskinesia (PCD) - is a disease caused by the body's cilia being immotile, dysmotile, or lacking altogether. As can be expected, most of its clinical symptoms come from the respiratory tracts, which are lined with ciliated epithelia. Thus sinusitis, rhinitis and bronchitis are likely to occur already from birth, otitis is nearly universal in childhood and bronchiectasis will often develop in the adults, unless they have been aggressively treated (Afzelius, 1976; Eliasson et al., 1977; Bush, 1998). A peculiar characteristic of the syndrome is its connection with the mechanism that regulates body laterality, thus whether it will be correctly or randomly determined (Afzelius, 1976). Random determination implies that equally many embryos will have a normal arrangement of the viscera, named situs solitus, or will have situs inversus. The combination of situs inversus, bronchiectasis and sinusitis is known under the name the Kartagener syndrome (Kartagener, 1933).

Immotile-cilia syndrome was initially based on four men only, but was assumed «not to be exceedingly rare» (Afzelius, 1976). Three of these men had situs inversus. The purpose of the present communication is to estimate the prevalence of immotile-cilia syndrome with or without the trait situs inversus and to determine its mode of inheritance. This was performed in various ways by collecting relevant information on the disease from as many persons with the syndrome as possible. Presently we know about 240 Swedish men, women and children having immotile-cilia syndrome. The data are compared to those of left-handedness.

\section{Prevalence of the immotile-cilia syndrome}

All Swedish citizens with diagnosed immotile-cilia syndrome and known to us, were listed in age order. The list contains no or few names for some years (e.g. those in the 1920s). Other years contain 9 or 10 names; these are the years when the search for patients was most intense. The 15 years 1976 to 1990 thus

Abbreviations used in this paper: PCD, primary ciliary dyskinesia.

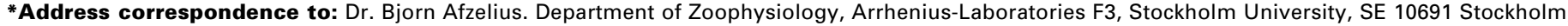
Sweden. Fax +468-156-756. e-mail: bjorn.afzelius@zub.su.se
} 
contain 69 names. During these 15 years 1,502,974 children were born in Sweden, which gives a value for the prevalence of immotile-cilia syndrome equaling 1 in 22,000.

The three years 1976, 1979, 1982 contained particularly many children with the syndrome: 26 from a total of 286, 409 children born. If these figures are representative, the prevalence would be 1 in 11,000 . It is however unlikely that all patients with the syndrome have been reported to us. The true figure is hence estimated to be not far from 1 in 10,000.

\section{Percentage of situs inversus in the syndrome}

Our list of Swedish citizens with immotile-cilia syndrome contains 239 names, 105 of which have situs inversus - this subgroup amounts to $44 \%$ of total. Most patients in the subgroup with situs inversus have the complete Kartagener syndrome, except children who have not developed bronchiectasis. The percentage values in the samples obtained from the 15 and 3 year-lists respectively amounts to 41 and $42 \%$ - values that are appreciably lower than $50 \%$, which would be expected in a random determination of the situs solitus versus situs inversus.

The lower than expected value of situs inversus is explained as follows. Immotile-cilia syndrome consists of about a dozen structurally distinct subgroups, one of which being characterized by the cilia lacking the two central microtubules - most cilia then have what is referred to as a $9+0$ axoneme rather than the normal $9+2$ one. Hence they resemble the recently described so-called nodal cilia, which have been recognized to be instrumental in determining the location of the forming heart and thereafter the direction of body asymmetry to be situs solitus (Nonaka et al., 2005). A mutation that implies the loss of the two central microtubules is likely to affect the respiratory $9+2$ cilia but not the nodal $9+0$ cilia. The corresponding patients will have the immotile-cilia syndrome but not coupled to situs inversus.

According to Jorissen et al. $(1997,2000)$ the $9+0$ mutant amounts to about $10 \%$ (or slightly less) of the total assembly. When this percentage is subtracted from the total number of affected persons (239 cases), one is left with 215 cases, half of which can be expected to have situs inversus. This figure - 108 is to be compared with the number obtained in our study, namely 105 cases. The prevalence of Kartagener syndrome hence is likely to be not far from 1 in 22,000.

\section{Percentage of left-handers in immotile-cilia syndrome}

Of the 112 persons with immotile-cilia syndrome who were asked about their handedness, 94 claimed themselves to be righthanders, 14 to be left-handers, whereas 4 considered themselves to be ambidextrous. There are thus $13 \%$ left-handers (disregarding the ambidextrous ones). The percentage left-handers in the group with situs inversus is slightly higher, namely 9 out of 63 ( $=14$ $\%$ ) but both values are similar to values in the general population (Gilbert and Wysocky, 1986; McManus 2002).

Left-handers in the older half of the cohort (born 1963 or earlier) were 6 out of 56 , thus $11 \%$, whereas in the younger half, persons born 1964 or later, were 8 out of $52=15 \%$ (again disregarding the four persons who claimed themselves to be ambidextrous).

\section{Some genetic data}

From a genetic point of view the data are of interest in two aspects. One refers to the trait situs inversus, which, as men- tioned, occurs in $50 \%$ of most - but not all - subgroups of the immotile-cilia syndrome. When both parents are healthy carriers, their children will have defective cilia in a 1:3 ratio, but have situs inversus in a 1:7 ratio. The latter ratio can be regarded as a case of inheritance of randomness (Layton, 1976; Afzelius, 1996).

The other aspect is the absence in the pedigrees of men with the syndrome who are fathers of sick or healthy children. Male fertility usually is low or nil when cilia are dysfunctional, as sperm flagella and cilia have nearly the same anatomy and composition. We have, however, not inquired about male fertility but are aware of a few studies where a man was found to have immotile respiratory cilia but motile spermatozoa and to be fertile.

Inquiring about female fertility is a less delicate task. Of 17 women who for some years have tried to become mothers, only 7 had been successful. Female fertility thus is somewhat lowered, maybe due to defective Fallopian or endometrial cilia.

In the present material 34 of a total of 168 sibs to a person with the immotile-cilia syndrome are similarly affected, thus $20 \%$. A somewhat higher figure, $25 \%$, would be expected. Many explanations for this discrepancy are conceivable, such as a higher prenatal or perinatal lethality in the immotile-cilia syndrome.

As in other genetic diseases, parents to the patients more often are closely related than are parents to persons in the general population. In our material, 8 out of 101 affected persons claimed that their parents are first or second cousins. This figure is about ten times higher than in the Scandinavian population in general.

From a mass roentgenographic study of the Norwegian population, Torgersen $(1947,1949)$ concluded that situs inversus has a prevalence of 1 in 8,000 . He further found that «bronchiectasis and nasal polyps occur in barely $10 \%$ of individuals with situs inversus». (Nasal polyps were nearly universal in the cohort with bronchiectasis.) This means that the prevalence of Kartagener syndrome in this material is about 1 in 80,000 and that of the immotile-cilia syndrome would be a little more than twice as common, thus probably about 1 in 36,000 . The study encompassed a third of the Norwegian population.

The values of prevalence of Kartagener syndrome and of immotile-cilia syndrome, found by us, are more than twice those given by Torgersen. It is unclear whether this difference is due to some technical factor, to improved diagnosis, or is a real one. Torgersen's data are from the first half of the $20^{\text {th }}$ century, whereas ours are from the second half. During the same period the prevalence of the other big asymmetry in human anatomy/ physiology has undergone a striking increase in the Western world - from about $5 \%$ to about $12 \%$ according to Gilbert and Wysocky (1986). A slight (although non-significant) increase in the prevalence of left-handers with time of birth is seen also in the present study, thus in patients with immotile-cilia syndrome.

McManus (2002) has analyzed the possible causes of the increase in left-handedness and concludes that at least some of the increase is due to a true change in the proportions of alleles responsible for left-handedness rather than to social pressure. With the same reasoning it could be concluded that the genes responsible for synthesis and/or motility in nodal cilia have decreased somewhat, resulting in a higher incidence of defective nodal cilia. A decrease in the gene for control of left-heartedness cannot be explained by social pressure and is unlikely to be due even to such environmental factors as an increased use of 

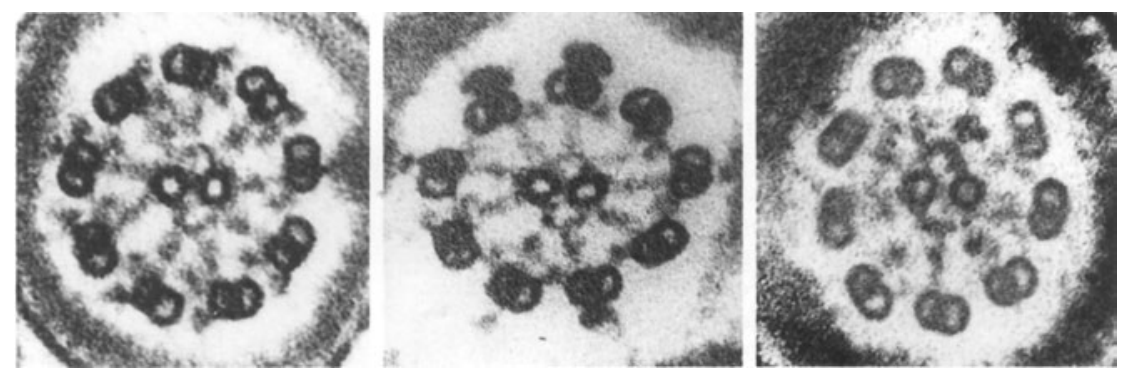

question rather than the resulting from a test battery. We are aware of the fact that this method has its limitations and gives values of handedness that are approximate only.

The senior author of this paper tried to get information from as many pediatricians, otologists, andrologists and pathologists as possible and from the Swedish Family Support Group of the disease. In positive cases the clinician asked the patient for permission to be contacted by the author, which in most cases was given. The author has also written several short papers on the syndrome in

Fig. 1. Cross-sectioned sperm tail flagella from a man with normally motile spermatozoa (left), from a man with dyskinetically motile spermatozoa (center) and from a man with immotile spermatozoa (right). The normally motile axoneme has inner and outer dynein arms, the dyskinetic axoneme has inner dynein arms only and the immotile axoneme lacks dynein arms.

ultrasound examinations - the time slot when the nodal cilia are active is a short and early one; it occurs about one week after conception.

Another, independent, functional asymmetry of the brain is that which can be detected with the so-called dichotomic listening test. In such a test eight out of nine examined persons with situs inversus were found to have what is called 'right ear advantage'; they reproduced sounds from the right ear more often than from the left ear (Tanaka et al., 1999). The percentage of right ear advantage was about the same as in the control group with situs solitus and the authors could conclude that the "functional asymmetry of the human brain must independently recognize laterality from situs asymmetry".

Immotile-cilia syndrome thus is inherited with what Layton called a «random determination of a developmental process» and so is left-handedness. In both cases, this term implies a choice between control (to right-handedness, to left-heartedness...) versus a loss of control, rather than a choice between right and left. The normal alleles will specify situs solitus and right-handedness. With a loss of control, right and left side will appear in equal numbers. The present study as well as previous ones show that the two major asymmetries of the human body are independently determined; for a divergent opinion see, however, McManus et al. (2004).

Whereas the mechanism of controlling sidedness of heart to the left is well understood (Nonaka et al., 2005), those of control of right-handedness or left-ear advantage are not. It is even difficult to figure out what kind of experimental animal model could be suitable for such studies.

\section{Experimental Procedures}

Immotile-cilia syndrome was diagnosed by severe problems from the respiratory tracts present already in early childhood together with either sperm or ciliary immotility (or dysmotility) or structural abnormalities in cilia and/or sperm tails as seen by electron microscopy. In a few cases defects in the ciliary escalator were detected by depositing a radioactive tracer in the lungs and measuring the speed of its transport (Eliasson et al., 1977). If the person to be examined - or his/her close relative presents the typical clinical picture and also has situs inversus, then he or she is considered to suffer from the immotile-cilia syndrome and no further tests are needed.

We have also collected data about right-handedness versus lefthandedness. Information was given to us as answers upon a direct medical colleagues).

Swedish popular science magazines, ladies magazines, weekly magazines, daily newspapers and then been contacted directly by persons who have the syndrome or believe so. (It might have been of advantage to perform such a study in a relatively small country and to be biologist rather than physician, hence not competing with

The second author has been responsible mainly for investigating suspected cases from children in Southern Sweden. We are both responsible for this study. As far as we understand the enquiries performed for this study entail no ethical problems.

\section{References}

AFZELIUS, B.A. (1976). A human syndrome caused by immotile cilia. Science193: 317-319.

AFZELIUS, B.A. (1996). Inheritance of randomness. Med. Hypoth. 43: 23-26.

BUSH, A., COLE, P., HARARI, M., et al. (1998). Primary ciliary dyskinesia. Diagnosis and standards of care. Eur. Resp. J. 12: 982-988.

ELIASSON, R., MOSSBERG, B., CAMNER, P., AFZELIUS., B.A. (1977). The immotile-cilia syndrome. New Engl. J. Med. 297: 1-6.

GILBERT, A.N. and WYSOCKY, C.J. (1986). Hand preference and age in the United States. Neuropsychologia 30: 601-608.

JORISSEN, M., BERTRAND, B., ELOY, P. (1997). Ciliary dyskinesia in the nose and paranasal sinuses. Acta Otorhinolar. Belg. 51: 353-366.

JORISSEN, M., WILLEMS, S., VAN DER SCHEUREN, B. et al. (2000). Ultrastructural expression of primary ciliary dyskinesia after ciliogenesis in culture. Acta Otorhinolar. Belg. 54: 343-366.

KARTAGENER, M. (1933). Zur Pathogenese der Bronchiektasien bei Situs viscerum inversus. Beitr. Klin. Tuberk, 83: 489-501.

LAYTON W.M (1976). Random inheritance of a developmental process. J. Hered. 67: 336-338.

McMANUS, C. (2002). Right hand, Left hand. Orion Books Ltd, London WC2H $9 E A$

McMANUS, C.I., MARTIN, N., STUBBINS,G.F., CHUNG, E.M.K., MITCHISON H.M. (2004). Handedness and situs inversus in primary ciliary dyskinesia. ProC. Biol. Sci. Ser B. 274: 2579-2582.

NONAKA, S., YOSHIBA S., WATANABE, R. (2005). De novo formation of left-right asymmetry by posterior tilt of nodal cilia. Plos Biology 3(8)e268.

TANAKA, S., KANAZAKI, R., YOSHIBAYASHI, M., KAMIYA.T., SUGISHITA, M. (1999). Dichotic listening in patients with situs inversus: brain asymmetry and situs asymmetry. Neuropsychologia 17: 869-874.

TORGERSEN, J. (1947). Transposition of viscera - bronchiectasis and nasal polyps. Acta Radiol. 38:17-24.

TORGERSEN, J. (1949) Genic factors in visceral asymmetry and in the development and pathological changes of lungs, heart and abdominal organs. Arch. Pathol. 47: 566-593.

Received: December 2005 Reviewed by Referees: February 2006 Modified by Authors and Accepted for Publication: March 2006 Published Online: May 2006 